\title{
Correction to: Effect of Age-Related Factors on the Pharmacokinetics of Lamotrigine and Potential Implications for Maintenance Dose Optimisation in Future Clinical Trials
}

\author{
Sven C. van Dijkman ${ }^{1}$ - Nico C. B. de Jager ${ }^{1}$ - Willem M. Rauwé ${ }^{1}$. \\ Meindert Danhof ${ }^{1} \cdot$ Oscar Della Pasqua ${ }^{1,2,3}$
}

Published online: 25 April 2018

(C) Springer International Publishing AG, part of Springer Nature 2018

\section{Correction to: Clin Pharmacokinet \\ https://doi.org/10.1007/s40262-017-0614-5}

Title which previously read

Effect of Age-Related Factors on the Pharmacokinetics of Lamotrigine and Potential Implications for Dose Optimisation in Epilepsy Patients

should read

Effect of Age-Related Factors on the Pharmacokinetics of Lamotrigine and Potential Implications for Maintenance Dose Optimisation in Future Clinical Trials

The original article can be found online at https://doi.org/10.1007/ s40262-017-0614-5.

Oscar Della Pasqua

oscar.e.della-pasqua@gsk.com

1 Division of Pharmacology, Leiden Academic Centre for Drug Research, Einsteinweg 55, 2333CC Leiden, The Netherlands

2 Clinical Pharmacology Modelling and Simulation, GlaxoSmithKline, Uxbridge UB11 1BT, UK

3 Clinical Pharmacology and Therapeutics Group, University College London, BMA House, Tavistock Square, London WC1H 9JP, UK
Abstract, Conclusion section, lines 4-8 which previously read

The availability of an integrated model including the whole patient population provides insight into the role of age-related changes in the disposition of lamotrigine, and potential implications for dose optimisation.

should read

The availability of an integrated model including the whole patient population provides insight into the role of age-related changes in the disposition of lamotrigine, and potential implications for maintenance dose optimisation in any future trials.

Table 4, heading which previously read

Optimised dosing regimens and predicted Css per age group

should read

Optimised maintenance dosing regimens and predicted steady-state concentration (Css) per age group in the simulated scenarios

Table 4, footnote which previously read

Each column summarises the proportion of patients in each group who are exposed above the toxicity level (20 $\mathrm{mg} / \mathrm{L})$, above the therapeutic maximum $(15 \mathrm{mg} / \mathrm{L})$, and below the therapeutic minimum $(2.5 \mathrm{mg} / \mathrm{L})$

$C_{s s}$ steady-state concentrations

should read

Each column summarises the proportion of patients in each group who are exposed above the toxicity level (20 
$\mathrm{mg} / \mathrm{L})$, above the therapeutic maximum $(15 \mathrm{mg} / \mathrm{L})$, and below the therapeutic minimum $(2.5 \mathrm{mg} / \mathrm{L})$

These scenarios do not include the initiation dose or titration steps, which are required to reduce the risk of serious cutaneous adverse reactions and account for interindividual differences in pharmacodynamics and safety

Section 4, para 8, lines 4-5 which previously read

...simulated regimens show that lamotrigine doses may be titrated at the onset of therapy...

should read

....simulated regimens show that lamotrigine doses should be titrated at the onset of therapy...

Section 4, para 8, lines 7-10 which previously read

In fact, understanding of the role of covariate factors can aid the clinician achieving a preset target concentration to a moderate degree.

should read

In fact, understanding of the role of covariate factors can aid investigators in achieving a pre-set target steady state concentration to a moderate degree, in proposed clinical trials.
Section 4, para 8, lines 13-15 which previously read

It can be anticipated that such a dosing algorithm may serve as a tool for clinicians at the start of treatment. Once a target maintenance dose is reached, model-guided dose adjustments can be made in conjunction with TDM sampling to further personalise therapy

should read

It can be anticipated that such a dosing algorithm may serve as a tool for investigators when developing their trial protocols. Once a target maintenance dose is reached, modelguided dose adjustments may be considered in conjunction with TDM sampling to further personalise therapy.

Section 5, para 1, lines 23-25 which previously read

These results should form the basis for the dose rationale in prospective clinical trials in infants and toddlers.

should read

These results should form the basis for the dose rationale in any future clinical trials in infants and toddlers. As the recommended low initiation dose, titration steps and interindividual differences in pharmacodynamics and safety were not considered here, they do not constitute recommendations for patients in clinical practice. 